\title{
Transfusion-Related Acute Lung Injured (TRALI): Current Concepts
}

\author{
P. Álvarez ${ }^{*}, 1,2$, R. Carrasco ${ }^{3}$, C. Romero-Dapueto ${ }^{4}$ and R.L. Castillo ${ }^{5}$ \\ ${ }^{1}$ Unidad de Paciente Crítico, Hospital Metropolitano La Florida, Santiago, Chile \\ ${ }^{2}$ Facultad de Medicina, Universidad Finis Terrae, Chile \\ ${ }^{3}$ Departamento de Medicina, Universidad de Chile, Hospital Salvador, Santiago, Chile \\ ${ }^{4}$ Servicio de Medicina Física y Rehabilitación, Clínica Alemana de Santiago, Santiago, Chile \\ ${ }^{5}$ Programa de Fisiopatología, Facultad de Medicina, Universidad de Chile, Santiago, Chile
}

\begin{abstract}
Transfusion-related acute lung injury (TRALI) is a life-threatening intervention that develops within 6 hours of transfusion of one or more units of blood, and is an important cause of morbidity and mortality resulting from transfusion. It is necessary to dismiss other causes of acute lung injury (ALI), like sepsis, acute cardiogenic edema, acute respiratory distress syndrome (ARDS) or bacterial infection. There are two mechanisms that lead to the development of this syndrome: immune-mediated and no immune- mediated TRALI. A common theme among the experimental TRALI models is the central importance of neutrophils in mediating the early immune response, and lung vascular injury. Central clinical symptoms are dyspnea, tachypnea, tachycardia, cyanosis and pulmonary secretions, altogether with other hemodynamic alterations, such as hypotension and fever. Complementary to these clinical findings, long-term validated animal models for TRALI should allow the determination of the cellular targets for TRALI-inducing alloantibodies as well as delineation of the underlying pathogenic molecular mechanisms, and key molecular mediators of the pathology. Diagnostic criteria have been established and preventive measures have been implemented. These actions have contributed to the reduction in the overalnumber of fatalities. However, TRALI still remains a clinical problem. Any complication suspected of TRALI should immediately be reported.
\end{abstract}

Keywords: Respiratory failure, transfusion-related acute lung injury.

\section{INTRODUCTION}

Transfusion Related Acute Lung Injury (TRALI) is defined as new ALI which develops within 6 hours of transfusion of one or more units, not attributable to another ALI risk factor. Amongst the numerous possible complications, TRALI has emerged as the most important cause of morbidity and mortality resulting from transfusion of blood or derived of the blood components. In fact, TRALI is currently being regarded as the first cause of severe morbidity and mortality related to blood transfusion therapy $[1,2]$.

The TRALI concept was described in 1985 by Popovsky, who published a trial on surgical patients, who developed hypotension, hypoxemia and respiratory failure, in the absence of hemodynamic overload from 1-6 hours after blood transfusion. A $72 \%$ of these patients required mechanical ventilation. There was a resolution of the condition to $96 \mathrm{~h}$ of onset of symptoms in $81 \%$ of cases. Moreover, the presence of leukocyte antibodies and anti-HLA type I was evidenced in a large percentage of donors [3].

*Address correspondence to this author at the Hospital Clínico Metropolitano La Florida; Facultad de Medicina, Universidad Finis Terrae. Av. Pedro de Valdivia 1509, Providencia, Santiago, Chile;

Tel: +56-224207100; Fax: +56-226121600;

E-mail: pe.alvarez.1@gmail.com
Two decades ago, TRALI was considered a rare complication of transfusion medicine. Nowadays, TRALI has emerged as the leading cause of transfusion-related mortality, presumably as a consequence of reaching international agreement on defining TRALI with subsequent increase recognition and reporting of TRALI cases [4]. The consensus definition in 2004 allowed a better estimate of TRALI, and possible TRALI for populations where other risk factors for acute lung injury are often present, mostly in critically ill patients. Of note, the incidence of TRALI is 50100 times higher in the critically ill than the general hospital population [2, 5]. In 2004, the consensus conference organized by the Canadian Blood Service and Hema-Quebec (Canada) established the criteria of the Canadian definition, which although very similar to those of the US consensus, introduced the difference between TRALI and possible TRALI, depending on whether or not a temporary relationship to other alternative risk factor for ALI production [6].

Both of them have limitations:

i) Only identify new and severe hypoxemia. The mild forms of TRALI may pass unidentified.

ii) TRALI diagnosis in patients with other ALI risk factors, requires expert clinical evaluation, and still there are indeterminate cases. 
iii) The definition does not include lab tests, so the diagnosis is only based on clinical variables.

iv) The limit of $6 \mathrm{~h}$ cannot lead to detect cases developed after this period.

Recently, Mark \& Corwin [7], have proposed an extension of the definition of TRALI in terms of the period in which the clinical manifestations begin: classic TRALI, which starts during the first 6 hours of transfusion and agrees with the model of immune TRALI, and late TRALI or TRALI-deferred, starting between 6 and $72 \mathrm{~h}$ of transfusion and whose mechanism of production would poorly defined mediators.

\section{PATHOPHYSIOLOGY OF TRALI}

Pathophysiology of TRALI is not fully understood at present. In the 1950s, initial reports were made on the role of antileukocyte antibodies in the pathogenesis of TRALI, when it was administered to a test subject, $50 \mathrm{ml}$ of blood from a patient containing leukoagglutinins. The clinical manifestations following this administration included hypotension, fever, respiratory failure and bilateral pulmonary infiltrates on chest, radiography with full recovery within 3 days.

There are two mechanisms, which lead to the development of the syndrome: immune-mediated and no immune- mediated TRALI.

i) Immune-mediated TRALI is caused by anti-HLA (human leukocyte antigen) antibodies class I, II and/or less frequent antibodies directed against specific antigens of granulocytes - HNA (human neutrophil antigen), which can be present in the serum of the recipient or donor, and react with the donor's or recipient's leukocytes, respectively $[8$, 9].

The Immune-mediated TRALI is estimated at $65-85 \%$ of all reported TRALI cases. Of antibody with specificity known, those directed against HNA-1a, HNA-1b, HNA-2a, HNA-3a (5b) and HLA-A2 are the most frequently documented [10]. Moreover, the presence of antibodies directed against HLA class II has been associated with TRALI.

An argument against immune model is that some patients who underwent the transfusion procedure did not develop TRALI, despite the presence of leukocyte antigens. Some arguments that can explain this issue are: i) heterozygosity antigen receptor recognized by the antibody; ii) the clinical presentation of transfused patient may predispose to a greater or lesser manifestation of pulmonary transfusion reaction, and iii) lack of detection by clinicians and lack of diagnosis of mild cases [11].

ii) Non-immune-mediated TRALI can be attributed to transfusion of biologically active compounds, which are accumulated in stored blood components such as bioactive lipids, proinflammatory cytokines or platelet microparticles with high procoagulant activity. In non-immune-mediated TRALI, no antibodies were detected despite the clinical symptoms [8].
The main inflammatory cells involved in TRALI pathogenesis are the polymorphonuclear neutrophils. For TRALI development, the production of leukocyte antibodies is crucial. There is a hypothesis of a "two-hit" model postulated by Silliman et al. 1995 [12], which assumes that TRALI onset depends on the coexistence of a factor that predisposes a recipient's reaction, as well as on the presence of leukocyte-activating compounds in transfused blood.

The "first hit" is mediated by any proinflammatory condition, it is an aggression that activates the pulmonary endothelium and promotes the recruitment and adhesion of neutrophils to the capillary endothelium, and later causes a disarray ofthe lung alveolar-capillary permeability barrier [13].

The "second hit" is induced by transfusion of a blood component. These compounds induce neutrophils activation and release of cytotoxic factors that cause endothelial damage and capillary injury. Reactive neutrophils secreteproinflammatory mediators such as cytokines (IL-1, IL-6, IL-8, TNF-alpha), release proteolytic enzymes (e.g. elastase, azurocidin), and produce reactive oxygen species $[8,12]$. These events initiate the cascade of immune reactions with the damage to the vascular endothelium, and produce pulmonary capillary fluid leakage in to the alveolar space, thus causing edema. The neutrophilic airway inflammation of the lung, and later the disruption of the lung alveolar-capillary permeability barrier, it seems to be similar to what is seen in other forms of ALI/ARDS, but with different origin [14].

There is a common pathway in the pathophysiology of TRALI, the damage to the alveolar-capillary membrane, where the neutrophil has been postulated as the protagonist cell for all reactions, and subsequently trigger as described above. Antibodies present in the transfused blood agglutinate to the first found neutrophils after transfusion, which are trapped in the microvasculature. It is well known that most of leukocyte antibodies of the IgG class, produce active neutrophils agglutination rather than passive. Neutrophils stimulated by leukocyte antibodies or biologically active lipids, liberate oxygen radicals which damage endothelial cells of the lung capillaries, leading to increased vascular permeability, and ultimately the passage of fluid and proteins into the alveoli [15].

The main pathophysiological events associated with TRALI are shown in Fig. (1).

\section{EXPERIMENTAL MODELS}

Animal models have disproved the microaggregate theory of acute lung injury from blood transfusions. The two major hypotheses of TRALI, passively transfused neutrophil and human leukocyte antigen antibodies, and biologically active lipids that accumulate in older, cellular blood products, have been replicated in animal models. In TRALIrelated events, the transfused products contain a stimulus that is insufficient to cause apulmonary transfusion reaction in the absence of one or more separate facilitating recipient factors, which can synergize with the transfused factors. 


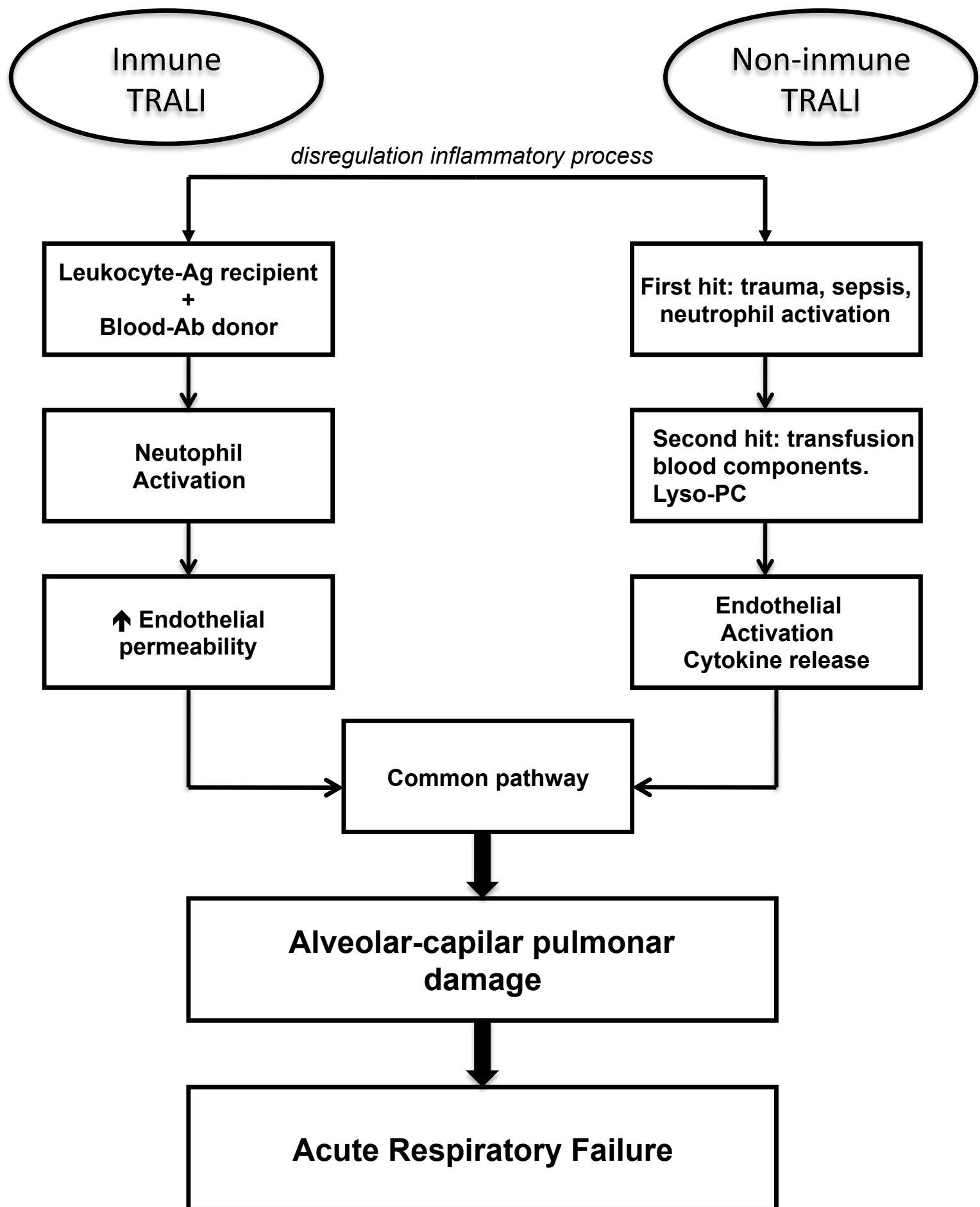

Fig. (1). Pathophysiology of TRALI. Ag, antigen; Ab, antibody; Lyso-PC, Lysophosphatidylcholines.

\subsection{Ex Vivo Lung Models of TRALI}

In this type of TRALI models, edema is produced by perfusate containing human neutrophils, human antigranulocyte alloantibody (anti-HNA 5b), and complement [16]. The lung edema resulted from an increase in vascular permeability and lung weight, which did not occur if the neutrophils of the perfusate were HNA 5b-negative or if any of the three components perfused was omitted. The HNA antibodies were also capable of direct neutrophil activation and reactive species oxygen (ROS) generation. Complement was not used, and the authors concluded that their model involves complement-independent antibody-induced neutrophil activation [17]. These data indicate that, direct antibodymediated activation of cognate antigen expressing neutrophils may be largely responsible for lung injury due to anti-HNA antibodies, but provide contradictory evidence regarding the role of complement. Other investigators used rat ex vivo lung explants to model the non-antibody-mediated TRALI [18]. They administered a nonlethal injection of LPS to the animals, followed by lung isolation and perfusion with 
plasma from either fresh or stored blood products. LPS injection and plasma perfusion constituted the first and second event, respectively. TRALI-like changes (pulmonary artery pressure, edema, mediator-release, ALI-histology) were observed with plasma from stored packed red blood cells or platelets, but not with fresh plasma [19]. In this model, the LPS-preprimed activity of endogenous neutrophils, wich was already present in the explanted lungs was key for inducing TRALI changes. On the other hand, the antibody-mediated the ex vivo lung model [20], the monoclonal anti-HNA-2a antibodies are capable of direct activation of the HNA-2a-expressing neutrophils.

\subsection{In Vivo Models of TRALI}

These models are scarce in the literature, since there is a high variability in the pathophysiological responses of animals. Indeed, considerable investment in "trial and error" based experimental approaches, may be needed in order to tease out the interacting variables that can produce TRALIlike reactions[21]. Some experimental variables are relevant in selected conditions for In vivo TRALI models: i) pharmacokinetic variables associated with TRALI-inducing agent, ii) induction of a "primed" state in the recipient, and iii) pathophysiological variables used for monitoring TRALI-events. The principal limitation of the developed TRALI models is related with pharmacological differences between the animals being used animals such as mice or rats, and the human physiology. In this case, the differential expression of leukocyte antigens has been described in rats and mice with TRALI or ARDS induced models [22].

The techniques for the development of ALI/ARDS in animal models, should also be applicable in modeling TRALI models. In particular, ALI/ARDS have been the central role of: i) activated neuthrophis, ii) activated pulmonary endothelium, iii) cell adhesion molecules on both these cells, as well as iv) a large amount of cytokines, v) other inflammatory mediators and vi) ROS [23].

\section{CLINICAL PRESENTATION}

The acute respiratory failure is the main finding in TRALI. The central clinical symptomis dyspnea and cooccurs with tachypnea, tachycardia, cyanosis and pulmonary secretions. Another hemodynamic alterations like fever, hypotension or hypertension (rare) are also reported [24]. All these clinical characteristics are observed during transfusion, or within $6 \mathrm{~h}$ of transfusion in the absence of other factors that can develop ALI, often during the first hour of a transfusion. During physical examination, the clinicians can find decreased breath sound, diffuse crackles over the lung fields, and shallow breathing. The chest radiography shows bilateral fluffy infiltrations with no cardiomegaly, and with this it can exclude cardiogenic edema. Hemodynamic monitoring helps to differentiate TRALI and cardiogenic pulmonary edema. Normal or low pulmonary capillary wedge pressure, and central venous pressure values are characteristic for TRALI $[12,25]$.

Actually, hemodynamic monitoring has been needed to exclude other causes of TRALI, because the symptoms are similar to other diseases, such as anaphylactic reaction, cardiogenic pulmonary edema, ARDS or bacterial infection, occasionally leukopenia, neutropenia, hypoalbuminemia and hypocomplementemia can be detected. The most prevalent symptom is a transient leukopenia, which arises in 5-35\% of patients [26].

\section{TREATMENT}

The treatment of TRALI is the support of lung function, and it does not differ from the treatment of ALI/ARDS of any other etiologies. Mild cases of ALI/ARDS could be treated with conventional measures such as oxygen therapy, and more serious cases require endotracheal intubation and mechanical ventilation strategies. The protective strategies should be applied in ALI/ ARDS, which aim to prevent the damage produced by mechanical lung ventilation [27, 28].

Sometimes, the presence of hemodynamic impairment requires the administration of fluids. Aggressive administration is not recommended, since as in the ALI/ARDS of other etiology, it has recently confirmed that a conservative strategy is accompanied by an improvement in pulmonary function with decreased in duration of mechanical ventilation and the ICU stay [28]. The lack of response to fluid therapy may force the introduction introducing of the inotropes. The use of corticosteroids has been stated in some case reports, but their use in TRALI is empirical and there is no evidence of their use [29].

\section{CONCLUDING REMARKS}

TRALI is a distinctive clinical entity or a syndrome of ALI/ARDS, it has increased in clinical importance in recent years. Long unrecognized, and existing as only scattered reports of non-cardiogenic pulmonary edema after transfusions, TRALI remained largely undiagnosed even after a case-series report. The relative contribution of biological response modifiers, and anti-leucocyte antibodies to clinical TRALI remains unclear, and it may be difficult to answer using animals and in vitro models. It seems likely that (i) traditional molecular mediators of inflammation are among such molecules critical for the manifestation of TRALI; (ii) the action of such mediators can be inhibited by appropriate antagonists; and (iii) results can be translated into therapeutic strategies for clinical use. The identification of risk factors further improves the risk-benefit assessment of a blood transfusion. Efforts to further decrease the risk of TRALI are needed to increase awareness of this syndrome among physicians.

\section{CONFLICT OF INTEREST}

The authors declare that there are no conflicts of interest.

\section{ACKNOWLEDGEMENTS}

Author's Role: All authors helped to write the manuscript and have seen and approved the final version.

\section{REFERENCES}

[1] Kleinman S, Caulfield T, Chan P, et al. Toward an understanding of transfusion-related acute lung injury: statement of a consensus panel. Transfusion 2004; 44: 1774-89. 
[2] Toy P, Popovsky MA, Abraham E, et al. Transfusion-related acute lung injury: Definition and review. Crit Care Med 2005; 33: 721-6.

[3] Popovsky MA, Moore SB. Diagnostic and pathogenetic considerations in transfusion-related acute lung injury. Transfusion 1985; 25: 573-7.

[4] Vlaar AP, Schultz MJ, Juffermans NP. Transfusion-related acute lung injury: a change of perspective. Neth J Med 2009; 67: 320-6.

[5] Kleinman S. A perspective on transfusion-related acute lung injury two years after the Canadian Consensus Conference. Transfusion 2006; 46: 1465-8

[6] Añón JM, García de Lorenzo A, Quintana M, et al. Transfusionrelated acute lung injury. Med Intensiva 2010; 34: 139-49.

[7] Marik PE, Corwin HL. Acute lung injury following to transfusion: expanding the definition. Crit Care Med 2008; 36: 3080-4.

[8] Swanson K, Dwyre DM, Krochmal J, Raife TJ. Transfusion-related acute lung injury (TRALI): current clinical and pathophysiologic considerations. Lung 2006; 184: 177-85.

[9] Müller MC, Juffermans NP. Transfusion-related acute lung injury: a preventable syndrome? Expert Rev Hematol 2012; 5: 97-106.

[10] Bierling P, Bux J, Curtis B, et al. Recommendations of the ISBT working party on granulocyte immunobiology for leucocyte antibody screening in the investigation and prevention of antibodymediated transfusion-related acute lung injury. Vox Sang 2009; 96: 266-9.

[11] Bux J. Transfusion-related acute lung injury (TRALI): a serious adverse event of blood transfusion. Vox Sang 2005; 89: 1-10.

[12] Silliman CC, Ambruso DR, Boshkov LK. Transfusion-related acute lung injury. Blood 2005; 105: 2266-73.

[13] Jaworski K, Maślanka K, Kosior DA. Transfusion-related acute lung injury: a dangerous and under diagnosed noncardiogenic pulmonary edema. Cardiol J 2013; 20: 337-44.

[14] Shaz BH. Giving TRALI the one-two punch. Blood 2012; 119: 1620-1.

[15] Bux J, Sachs U. The pathogenesis of transfusion-related acute lung injury (TRALI). Br J Haematol 2007; 136: 788-99.

[16] Seeger W, Schneider U, Kreusler B, et al. Reproduction of transfusion-related acute lung injury in an ex vivo lung model. Blood 1990; 76: 1438-44.
[17] Sachs UJ, Hattar K, Weissmann N, et al. Antibody-induced neutrophil activation as a trigger for transfusion-related acute lung injury in an ex vivo rat model. Blood 2006; 107: 1217-19.

[18] Silliman CC, Bjornsen AJ, Wyman TH, et al. Plasma and lipids from stored platelets cause acute lung injury in an animal model. Transfusion 2003; 43: 633-40.

[19] Lögdberg LE, Vikulina T, Zimring JC, Hillyer CD. Animal models of transfusion-related acute lung injury. Transfus Med Rev 2009; 23: 13-24.

[20] Bux J, Becker F, Seeger W, et al: Transfusion-related acute lung injury due to HLA-A2-specific antibodies in recipient and NB1specific antibodies in donor blood. Br J Haematol 1996; 93: $707-$ 13.

[21] Vlaar AP, Zweers MM, Schultz MJ, et al. Developing specific therapeutic strategies for transfusion-related acute lung injury: an overview of potentially useful animal models. Cardiovasc Hematol Agents Med Chem 2007; 5: 319-26.

[22] Woźniak MJ1, Bowring C, Lucas G, Ridgwell K. Detection of HNA-3a and $-3 b$ antibodies using transfected cell lines and recombinant proteins. Transfusion 2012; 52: 1458-67.

[23] Ritter C, da Cunha AA, Echer IC, et al. Effects of N-acetylcysteine plus deferoxamine in lipopolysaccharide induced acute lung injury in the rat. Crit Care Med 2006; 34: 471-7.

[24] Aravinthan A, Sen S, Marcus N. Transfusion-related acute lung injury: a rare and life-threatening complication of a common procedure. Clin Med 2009; 9: 87-9.

[25] Triulzi DJ. Transfusion-related acute lung injury: an update. Hematol Am Soc Hematol Educ Program 2006: 497-501.

[26] Fadeyi EA, De Los Angeles MM, Wayen AS, et al. The transfusion of neutrophil-specific antibodies causes leukopenia and a broad spectrum of pulmonary reactions. Transfusion 2007; 47: 545-50.

[27] Gordo Vidal F, Delgado Arnaiz C, CalvoHerranz E. Mechanical ventilation induced lung injury. Med Intensiva 2007; 31: 18-26.

[28] Vlaar AP, Juffermans NP. Transfusion-related acute lung injury: a clinical review. Lancet 2013; 382: 984-94.

[29] Moore SB. Transfusion-related acute lung injury (TRALI): clinical presentation, treatment, and prognosis. Crit Care Med 2006; 34:S114-7.

(C) Álvarez et al.; Licensee Bentham Open.

This is an open access article licensed under the terms of the Creative Commons Attribution Non-Commercial License (http://creativecommons.org/licenses/by-nc/3.0/) which permits unrestricted, non-commercial use, distribution and reproduction in any medium, provided the work is properly cited. 